\title{
Steady-State Analysis of SECIR Rumor Spreading Model in Complex Networks
}

\section{Yujiang Liu, Chunmei Zeng, Youquan Luo}

Key Laboratory of Jiangxi Province for Numerical Simulation and Emulation Techniques, Gannan Normal University, Ganzhou, China

Email: gnsylyj@126.com

How to cite this paper: Liu, Y.J., Zeng, C.M. and Luo, Y.Q. (2019) Steady-State Analysis of SECIR Rumor Spreading Model in Complex Networks. Applied Mathematics, 10, 75-86.

https://doi.org/10.4236/am.2019.103007

Received: February 19, 2019

Accepted: March 18, 2019

Published: March 21, 2019

Copyright $\odot 2019$ by author(s) and Scientific Research Publishing Inc. This work is licensed under the Creative Commons Attribution International License (CC BY 4.0).

http://creativecommons.org/licenses/by/4.0/

\begin{abstract}
In this paper, the SECIR rumor spreading model is formulated and analyzed, in which the social education level and the counterattack mechanism are taken into consideration. The results show that improving education level and increasing the ratio of counter are effective in reducing the risk of rumor propagation and enhancing the resistance to rumor propagation.
\end{abstract}

\section{Keywords}

SECIR Model, Rumor Spreading, Homogeneous Network, Counterattack Mechanism

\section{Introduction}

Rumor is a kind of social phenomenon that an unverified account or explanation of events spreads on a large-scale in a short time through people's communication [1] [2] [3]. The spread of rumor can manipulate the public opinion in a locality, even can cause panic in some of the important public event [4] [5] [6] [7] [8]. Today, the increasing prevalence of social networking services, rumors spread by twitters, blogs, microblogs, WeChat and so on. In the Internet, the spreading of rumor is similar to epidemic spreading, but rumor's spreading quantitative models have been rather limited in the complex network.

The standard model of rumor spreading is the Daley-Kendal (DK) model [9] [10]. The population in a local area is grouped ignorants, spreaders and stiflers. In this model, the rumor is propagated through the pair-wise contacts between spreaders and the others in the population. Spreader attempts to "infect" the other individuals with rumor. In the contact, ignorance becomes spreader, the others of individuals become stiflers. In the Maki-Thompson (MK) model [11] as the DK model's variant, the spreader becomes stifler only who is the initiating 
spreader. The deficiencies of the DK model are considering homogeneous topology, and the simplified topology may not adequately describe the rumor's spreading process in the Internet [12]-[17]. Zanette [18] [19] analyzed the MK model on a small-world network. His studies show that rumor "dies" in a small scale of its origin with varying network randomness. Morno et al. [20] [21] considered a rumor spreading model on a scale-free network. The results are the uniformity of the network which has a great impact on the rumor's spreading process. Nekovee et al. [22] introduced the SIR model with forgetting mechanism and derived mean-field equation that describes the dynamics of rumor spreading process. His studies show that the SIR model is suitable for chain emails and large-scale information dissemination algorithms on the Internet. Zhao et al. [23] [24] refined the SIR rumor spreading model and took into account the remembering mechanism in addition to the forgetting mechanism. Zan et al. [25] considered the counterattack mechanism in the SIR model and introduced two models: Susceptible-infective-counterattack-Refractory (SICR) model and adjusted-SICR model. They derived mean-field equations to describe its dynamics in homogeneous network and involve steady-state analysis. Their studies show the self-resistance characteristic of networks to a rumor. Afassinou [26] extended the SIR model with the forgetting mechanism and population's education rate and introduced SEIR model. He distinguishes two types of individuals in a population: educated individuals and non-educated individuals. His results show that improving the education rate of the population catalyzes the rumor spreading termination process. In social networks, when people with a higher degree of education heard a rumor which is in serious conflict with his/her belief, he/she is easier to counterattack the rumor, and even do the best to prevent the rumor propagation.

In this paper, inspiring of Zan et al. [25], we consider two influential factors in rumor spreading process: the population's education rate and the self-resistance feature of network. Motivatedly, we extend rumor spreading model-the SECIR model.

The remaining part of the paper is organized as follows. We formulate the propagation mechanism of the SECIR model in a social network, and derive a system of nonlinear ordinary differential equations that describe dynamics of rumor spreading process in Section 2. In Section 3, we analyze the steady-state of the SECIR model. We give some of the conclusions in the last section.

\section{Model}

We study the SECIR model in a closed homogeneously mixed population that we differentiate into five distinct classes: the rumor-mongers (spreader, S), those who are spreading the rumor, the non-educated ignorants individuals class (Ignorant, I), the people who never heard the rumor, the educated ignorants individuals class (Educatee, E), the people who never heard the rumor, but have more sophisticated behaviours with the non-educated ignorant individuals when they encountered the spreader, the counterattack class (Counter, C), those who 
do not agree but refute the rumor, and persuade others to agree with him (refute the rumor), and the stiflers class (Recovered, $\mathrm{R}$ ), the ones who heard the rumor but have lost interest in disseminating it. For simplicity, we refer to the rumor spreading model as the SECIR model.

According to the MK model, we assume the rumor spreads by directed contact of the spreads with others in the population, and the contacts between rumor-mongers and the rest of the population are governed by the following dynamics (As shown in Figure 1):

- Whenever a non-educated ignorant contact a spreader, the ignorant will be a relatively large probability $\left(\beta_{1}\right)$ into the spreader, but also with a smaller probability $\left(\alpha_{1}\right)$ of transition to a stifler;

- Whenever an educated ignorant contact a spreader, the ignorant will be one of the three class: spreader or stifler analog the non-educated ignorant, and he can be evolved into counterattack, and the change probability is $\beta_{2}, \alpha_{2}$ and $\theta$, respectively;

- Whenever a spreader contact a spreader, a stifler or a counter, the spreader will be into stifler with the probability $g, g$ and $\eta$, respectively;

Let $I(t), E(t), C(t), C(t)$, and $R(t)$ respectively represent the density of the corresponding compartment in the total population. Namely that we have $I(t)+E(t)+C(t)+S(t)+R(t)=1$. Note that the resistance to the rumor of the educated ignorants more than the non-educated ignorants, we assume that

$$
\beta_{1}>\beta_{2}, \alpha_{1}>\alpha_{2} \text { and } \eta>g \text {. }
$$

In accordance with the above rules, the mean-field equations of the SECIR model can be described as follows:

$$
\begin{aligned}
& \frac{\mathrm{d} I(t)}{\mathrm{d} t}=-\left(\beta_{1}+\alpha_{1}\right) \bar{k} I(t) S(t) \\
& \frac{\mathrm{d} E(t)}{\mathrm{d} t}=-\left(\beta_{2}+\alpha_{2}+\theta\right) \bar{k} E(t) S(t) \\
& \frac{\mathrm{d} C(t)}{\mathrm{d} t}=\theta \bar{k} E(t) S(t) \\
& \frac{\mathrm{d} S(t)}{\mathrm{d} t}=\beta_{1} \bar{k} I(t) S(t)+\beta_{2} \bar{k} E(t) S(t)-g \bar{k} S(t)(S(t)+R(t))-\eta \bar{k} S(t) C(t) \\
& \frac{\mathrm{d} R(t)}{\mathrm{d} t}=\alpha_{1} \bar{k} I(t) S(t)+\alpha_{2} \bar{k} E(t) S(t)+g \bar{k} S(t)(S(t)+R(t))+\eta \bar{k} S(t) C(t)
\end{aligned}
$$

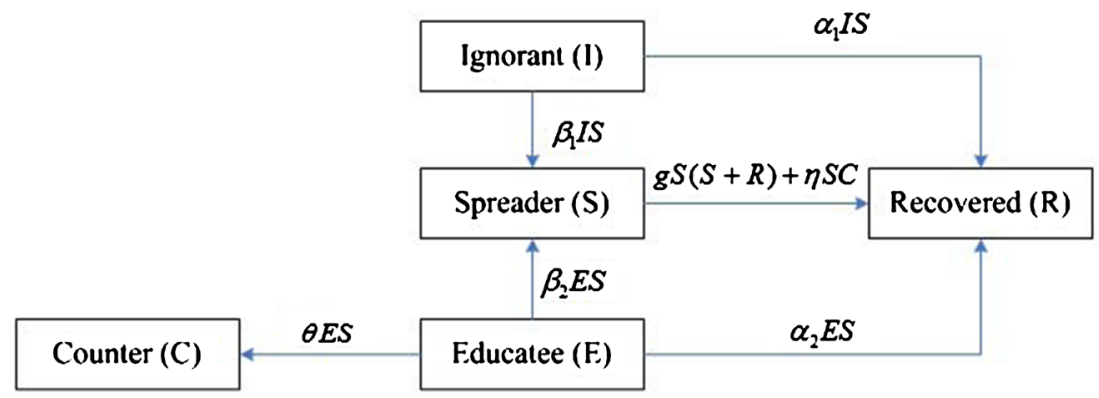

Figure 1. SECIR rumor spreading model. 
We assume that all in the population are $I$ or $E$ but only one spreader at the beginning of the rumor spreading, and the ratio of $E$ to the sum of $I$ and $E$ is $\epsilon$ when $t=0$. Namely, when $t=0$, the initial condition of rumor spreading is given as follows:

$$
I(0)=\frac{N-1}{N}(1-\epsilon), E(0)=\frac{N-1}{N} \epsilon, S(0)=\frac{1}{N}, C(0)=0, R(0)=0 .
$$

Note that for an ignorant, he/she can be a spreader or stifler, so we have $\beta_{1}+\alpha_{1}<1$, and $1-\beta_{1}-\alpha_{1}$ is the probability that no one tells him/her the rumor. For the same reason, $\beta_{2}+\alpha_{2}+\theta<1$ is considered.

\section{Model Analysis}

We then postulate that the number of individuals in the $I$ class that have heard about the rumor is the same as the number of individuals in the $E$ class that have heard about the rumor. This simply translates to

$$
\begin{aligned}
\beta_{1}+\alpha_{1} & =\beta_{2}+\alpha_{2}+\theta \\
& =M
\end{aligned}
$$

From the first and the second equation of (2), we have

$$
\frac{\mathrm{d} E(t)}{\mathrm{d} I(t)}=\frac{E(t)}{I(t)}
$$

Solve the above differential equation with the initial conditions (3), we obtain

$$
\begin{aligned}
\frac{E(t)}{I(t)} & =\frac{E(0)}{I(0)} \\
& =\frac{\epsilon}{1-\epsilon}
\end{aligned}
$$

From the first and the third Equation (2), we obtain

$$
\frac{\mathrm{d} C(t)}{\mathrm{d} t}=-\frac{\theta}{\alpha_{2}+\beta_{2}+\theta} \frac{\mathrm{d} E(t)}{\mathrm{d} t}
$$

With the initial conditions (3), we can derive the relational expression between $C(t)$ and $E(t)$ by separation of variable,

$$
C(t)=\frac{\theta}{\alpha_{2}+\beta_{2}+\theta}(\epsilon-E(t))
$$

From (6) and (7), we have

$$
\begin{aligned}
S(t)+R(t) & =1-(I(t)+C(t)+E(t)) \\
& =1-\frac{1-\epsilon}{\epsilon} E(t)-\frac{\theta}{\alpha_{2}+\beta_{2}+\theta}(\epsilon-E(t))-E(t) \\
& =\left(1-\frac{\theta \epsilon}{\alpha_{2}+\beta_{2}+\theta}\right)-\left(\frac{1}{\epsilon}-\frac{\theta}{\alpha_{2}+\beta_{2}+\theta}\right) E(t)
\end{aligned}
$$

Note that $\tau=\frac{g}{\epsilon}+\frac{(\eta-g) \theta}{\alpha_{2}+\beta_{2}+\theta}$ and $\beta=\frac{1-\epsilon}{\epsilon} \beta_{1}+\beta_{2}$, then we have 


$$
\begin{aligned}
\frac{\mathrm{d} S(t)}{\mathrm{d} t}= & \beta_{1} \bar{k} I(t) S(t)+\beta_{2} \bar{k} E(t) S(t)-g \bar{k} S(t)(S(t)+R(t))-\eta \bar{k} S(t) C(t) \\
= & \beta_{1} \bar{k} \frac{1-\epsilon}{\epsilon} E(t) S(t)+\beta_{2} \bar{k} E(t) S(t)-\eta \bar{k} S(t) \frac{\theta}{\alpha_{2}+\beta_{2}+\theta}(\epsilon-E(t)) \\
& -g \bar{k} S(t)\left(\left(1-\frac{\theta \epsilon}{\alpha_{2}+\beta_{2}+\theta}\right)-\left(\frac{1}{\epsilon}-\frac{\theta}{\alpha_{2}+\beta_{2}+\theta}\right) E(t)\right) \\
= & \left(\frac{1-\epsilon}{\epsilon} \beta_{1}+\beta_{2}+\frac{g}{\epsilon}+\frac{(\eta-g) \theta}{\alpha_{2}+\beta_{2}+\theta}\right) \bar{k} E(t) S(t)-\left(g+\frac{\theta(\eta-g) \epsilon}{\alpha_{2}+\beta_{2}+\theta}\right) \bar{k} S(t) \\
= & (\beta+\tau) \bar{k} E(t) S(t)-\epsilon \tau \bar{k} S(t)
\end{aligned}
$$

From the second equation of (2), therefore (9) becomes

$$
\frac{\mathrm{d} S(t)}{\mathrm{d} t}=\frac{\frac{\epsilon \tau}{E(t)}-(\beta+\tau)}{M} \frac{\mathrm{d} E(t)}{\mathrm{d} t}
$$

Solving the differential equations above by the method of separation of variables, we have

$$
S(t)=\frac{\epsilon \tau}{M}(\ln E(t)-\ln \epsilon)-\frac{\beta+\tau}{M}(E(t)-\epsilon)
$$

By the second equation of (2), it is easy to see that $\frac{\mathrm{d} E(t)}{\mathrm{d} t}<0$ and $E(t)$ are monotonically decreasing and continuous function. Let $\frac{\mathrm{d} S(t)}{\mathrm{d} t}=0$, we obtain $E(t)=\frac{\epsilon \tau}{\beta+\tau}$. It is easy to see that, the peak value of spreader is

$$
S_{\max }=\frac{\epsilon \beta}{M}+\frac{\epsilon \tau}{M} \ln \left(\frac{\tau}{\beta+\tau}\right)
$$

$$
\begin{aligned}
\frac{\mathrm{d} R(t)}{\mathrm{d} t}= & \alpha_{1} \bar{k} I(t) S(t)+\alpha_{2} \bar{k} E(t) S(t)+g \bar{k} S(t)(S(t)+R(t))+\eta \bar{k} S(t) C(t) \\
= & \alpha_{1} \bar{k} \frac{1-\epsilon}{\epsilon} E(t) S(t)+\alpha_{2} \bar{k} E(t) S(t)+\eta \bar{k} S(t) \frac{\theta}{\alpha_{2}+\beta_{2}+\theta}(\epsilon-E(t)) \\
& +g \bar{k} S(t)\left(\left(1-\frac{\theta \epsilon}{\alpha_{2}+\beta_{2}+\theta}\right)-\left(\frac{1}{\epsilon}-\frac{\theta}{\alpha_{2}+\beta_{2}+\theta}\right) E(t)\right) \\
= & \left(\frac{1-\epsilon}{\epsilon} \alpha_{1}+\alpha_{2}-\frac{g}{\epsilon}-\frac{(\eta-g) \theta}{\alpha_{2}+\beta_{2}+\theta}\right) \bar{k} E(t) S(t)+\left(g+\frac{\theta(\eta-g) \epsilon}{\alpha_{2}+\beta_{2}+\theta}\right) \bar{k} S(t) \\
= & (\alpha-\tau) \bar{k} E(t) S(t)+\epsilon \tau \bar{k} S(t)
\end{aligned}
$$

where $\alpha=\frac{1-\epsilon}{\epsilon} \alpha_{1}+\alpha_{2}$. From the second equation of (2), therefore (12) becomes

$$
\frac{\mathrm{d} R(t)}{\mathrm{d} t}=\left(\frac{\tau-\alpha}{\alpha_{2}+\beta_{2}+\theta}-\frac{\epsilon \tau}{\alpha_{2}+\beta_{2}+\theta} \frac{1}{E(t)}\right) \frac{\mathrm{d} E(t)}{\mathrm{d} t}
$$

Solving the above differential equations, we get 


$$
R(t)=\frac{\alpha-\tau}{\alpha_{2}+\beta_{2}+\theta}(\epsilon-E(t))+\frac{\epsilon \tau}{\alpha_{2}+\beta_{2}+\theta}(\ln \epsilon-\ln E(t))
$$

It is easy know that $S_{\infty}=0$, let $t \rightarrow \infty$, then (8) becomes

$$
R_{\infty}=\left(\frac{1}{\epsilon}-\frac{\theta}{\alpha_{2}+\beta_{2}+\theta}\right)\left(\epsilon-E_{\infty}\right)
$$

Let $t \rightarrow \infty$, Substituting (15) into (14), it becomes

$$
\begin{gathered}
\left(\frac{1}{\epsilon}-\frac{\alpha-\tau+\theta}{\alpha_{2}+\beta_{2}+\theta}\right)\left(\epsilon-E_{\infty}\right)=\frac{\epsilon \tau}{\alpha_{2}+\beta_{2}+\theta}\left(\ln \epsilon-\ln E_{\infty}\right) \\
\alpha+\beta=\frac{1-\epsilon}{\epsilon}\left(\beta_{1}+\alpha_{1}\right)+\left(\beta_{2}+\alpha_{2}\right) \\
=\frac{1-\epsilon}{\epsilon}\left(\beta_{2}+\alpha_{2}\right)+\left(\beta_{2}+\alpha_{2}\right) \\
=\frac{1}{\epsilon}\left(\beta_{2}+\alpha_{2}+\theta\right)-\theta
\end{gathered}
$$

Solve from (17), we get $\epsilon=\frac{\beta_{2}+\alpha_{2}+\theta}{\beta+\alpha+\theta}$, substitute into (16), we have

$$
\left(\epsilon-E_{\infty}\right)=\frac{\epsilon \tau}{\beta+\tau}\left(\ln \epsilon-\ln E_{\infty}\right)
$$

Let $A(t)=I(t)+E(t)=\frac{1-\epsilon}{\epsilon} E(t)+E(t)=E(t) / \epsilon$, from (18) we get the final size

$$
A_{\infty}=\frac{\tau}{\beta+\tau} \ln A_{\infty}+1
$$

Theorem 1. For $0<\sigma<1$, the equation $x=\sigma \ln x+1$ has two solutions, $x=1$ and a nontrivial solution $x_{1}$, where $0<x_{1}<\sigma$.

Proof. Obviously $x=1$ is a solution of $x=\sigma \ln x+1$.

Let $f(x)=x-\sigma \ln x-1$, and take the derivative of $f(x)$ with respect to $x$. $f^{\prime}(x)=1-\sigma / x, f^{\prime \prime}(x)=\sigma / x^{2}>0$.

Let $f^{\prime}(x)=0$, we obtain the unique minimum point $x=\sigma$, and the function $f(x)$ is a convex function, we have $f(\sigma)=\sigma-\sigma \ln \sigma-1<\sigma+\sigma(1 / \sigma-1)-1=0$, and $f\left(0^{+}\right)=\infty$. According to the Mean Value Theorem, $f(x)$ have a nontrivial solution $x_{1}$, where $0<x_{1}<\sigma$.

Theorem 2. If the parameters are satisfied (1), (3) and (4), we have

(1) $S_{\max }$ is decrease with $\epsilon$, if the other parameters keep constant.

(2) $S_{\max }$ is increase with $\beta_{1}, \beta_{2}$ but decrease with $\alpha_{1}, \alpha_{2}$, if the other parameters keep constant.

(3) $S_{\max }$ is decrease with $g, \eta$, if the other parameters keep constant.

Proof. (1) From (11), differential with $\epsilon$, we have

$$
\begin{aligned}
\frac{\mathrm{d} S_{\max }}{\mathrm{d} \epsilon} & =-\frac{\beta_{1}-\beta_{2}}{M}+\frac{\epsilon \tau}{M}\left(\ln \frac{\tau}{\beta+\tau}\right)_{\epsilon}+\frac{(\epsilon \tau)_{\epsilon}^{\prime}}{M} \ln \frac{\tau}{\beta+\tau} \\
& =-\frac{\beta_{1}-\beta_{2}}{M}+\frac{\epsilon \tau}{M}\left(\frac{-\beta g+\tau \beta_{1}}{M \epsilon(\beta+\tau)}\right)+\frac{(\eta-g) \theta}{M^{2}} \ln \frac{\tau}{\beta+\tau}
\end{aligned}
$$




$$
\begin{aligned}
& \leq-\frac{\beta_{1}-\beta_{2}}{M}+\frac{\epsilon \tau}{M}\left(\frac{-\beta g+\tau \beta_{1}}{M \epsilon(\beta+\tau)}\right)+\frac{(\eta-g) \theta}{M^{2}}\left(\frac{\tau}{\beta+\tau}-1\right) \\
& =-\frac{\left((1-\epsilon) \beta_{1}+\beta_{2} \epsilon\right)\left(\beta_{1}-\beta_{2}\right)}{M(\beta+\tau) \epsilon}
\end{aligned}
$$

Therefore, $\frac{\mathrm{d} S_{\max }}{\mathrm{d} \epsilon}<0$, which implies that $S_{\max }$ decreases as $\epsilon$ increases.

(2) Since that $\tau=\frac{g}{\epsilon}+\frac{(\eta-g) \theta}{\alpha_{2}+\beta_{2}+\theta}$ and $\beta=\frac{1-\epsilon}{\epsilon} \beta_{1}+\beta_{2}$, we have $\tau_{\beta_{i}}^{\prime}=0$, $\tau_{\alpha_{i}}^{\prime}=0, \quad i=1,2, \beta_{\beta_{i}}^{\prime}>0, \beta_{\alpha_{i}}^{\prime}<0$. Taking the derivative of (11), with respect to $\beta_{i}$, we have $\frac{\mathrm{d} S_{\max }}{\mathrm{d} \beta_{i}}=\frac{\epsilon}{M} \beta_{\beta_{i}}^{\prime}-\frac{\epsilon \tau}{M} \frac{\beta_{\beta_{i}}^{\prime}}{\beta+\tau}=\frac{\epsilon}{M} \frac{\beta}{\beta+\epsilon} \beta_{\beta_{i}}^{\prime}>0$. It is easy to see that $S_{\max }$ increases as $\beta_{i}(i=1,2)$ increases.

By the same way, we can proof that $S_{\max }$ is decrease with $\alpha_{1}, \alpha_{2}$.

(3) Similarly (2), we have $\beta_{\eta}^{\prime}=0, \beta_{g}^{\prime}=0, \tau_{x}^{\prime}>0,(x=\eta$ or $g)$, then

$$
\begin{aligned}
\frac{\mathrm{d} S_{\max }}{\mathrm{d} x} & =\frac{\epsilon}{M} \ln \frac{\tau}{\beta+\tau} \tau_{x}^{\prime}+\frac{\epsilon \tau}{M}\left(\frac{\tau_{x}^{\prime}}{\tau}-\frac{\tau_{x}^{\prime}}{\beta+\tau}\right) \\
& =\left(\frac{\epsilon}{M} \ln \frac{\tau}{\beta+\tau}+\frac{\epsilon \beta}{M(\beta+\tau)}\right) \tau_{x}^{\prime} \\
& <\left(\frac{\epsilon}{M}\left(\frac{\tau}{\beta+\tau}-1\right)+\frac{\epsilon \beta}{M(\beta+\tau)}\right) \tau_{x}^{\prime} \\
& =0
\end{aligned}
$$

which means that $S_{\max }$ is decrease with $g, \eta$ The proof is complete.

Theorem 3. If the parameters are satisfied (1), (3) and (4), we have

(1) the other parameters keep constant, the final state $A_{\infty}$ is increased with $\epsilon$.

(2) the other parameters keep constant, the final state $A_{\infty}$ is decreased with $\beta_{1}, \beta_{2}$ but decrease with $\alpha_{1}, \alpha_{2}$.

(3) the other parameters keep constant, the final state $A_{\infty}$ is increased with $g, \eta$. Proof. Let $\sigma=\frac{\tau}{\beta+\tau}$, from (19) we have

$$
\frac{\mathrm{d} A_{\infty}}{\mathrm{d} \chi}=\frac{\mathrm{d} \sigma}{\mathrm{d} \chi} \ln A_{\infty}+\sigma \frac{1}{A_{\infty}} \frac{\mathrm{d} A_{\infty}}{\mathrm{d} \chi}=\frac{A_{\infty} \ln A_{\infty}}{A_{\infty}-\sigma} \frac{\mathrm{d} \sigma}{\mathrm{d} \chi}
$$

From Theorem $1,0<A_{\infty}<\sigma$, it is obviously that $\frac{A_{\infty} \ln A_{\infty}}{A_{\infty}-\sigma}>0$, so, if we know that the plus-minus sign of $\frac{\mathrm{d} \sigma}{\mathrm{d} \chi}$, then the plus-minus sign of $\frac{\mathrm{d} A_{\infty}}{\mathrm{d} \chi}$ can be determined, and furthermore, the monotonicity between $A_{\infty}$ and parameters $\chi$ can be proved.

(1)

$$
\frac{\mathrm{d} \sigma}{\mathrm{d} \epsilon}=\frac{\tau_{\epsilon}^{\prime} \beta-\tau \beta_{\epsilon}^{\prime}}{(\beta+\tau)^{2}}=\frac{\beta_{1} \tau-g \beta}{\epsilon^{2}(\beta+\tau)^{2}}=\frac{\left(\beta_{1}-\beta_{2}\right) g+\frac{(\eta-g) \theta}{M} \beta_{1}}{\epsilon^{2}(\beta+\tau)^{2}}
$$


so $\frac{\mathrm{d} \sigma}{\mathrm{d} \epsilon}>0$, that is, $\sigma$ increases as $\epsilon$ increases, and also implies that $A_{\infty}$ increases as $\epsilon$ increases.

(2)

$$
\frac{\mathrm{d} \sigma}{\mathrm{d} \beta_{i}}=-\frac{\tau \beta_{\beta_{i}}^{\prime}}{(\beta+\tau)^{2}}<0,(i=1,2)
$$

similarly, we can get $\frac{\mathrm{d} \sigma}{\mathrm{d} \alpha_{i}}>0$. and so $\frac{A_{\infty}}{\mathrm{d} \beta_{i}}<0, \frac{A_{\infty}}{\mathrm{d} \alpha_{i}}<0$, where $i=1,2$, which proves (2).

(3)

$$
\frac{\mathrm{d} \sigma}{\mathrm{d} x}=\frac{\tau_{x}^{\prime} \beta}{(\beta+\tau)^{2}}, x \in\{\eta, g\}
$$

then, $\frac{\mathrm{d} \sigma}{\mathrm{d} x}>0$, it means that $A_{\infty}$ increases as $\eta(g)$ increases. The proof is complete.

\section{Numerical Simulation}

We assume $N=10^{5}$, the average degree of network $\bar{k}=10$, and the initial condition of the model follows equation of (2).

Figure 2 shows the general trends of the five kinds of agents in the SEICR rumor spreading model. We see the density of spreaders begin to expand rapidly from the initial rumor spread. As the rumor spread further, the density of spreaders reaches a peak and thereafter declines. Finally the density of spreaders is to zero and this leads to the termination of rumor spreading. And over the course of the rumor spreading, the density of ignorants and educatees always decreases, and finally evolves to zero. but the density of counter and the recovered

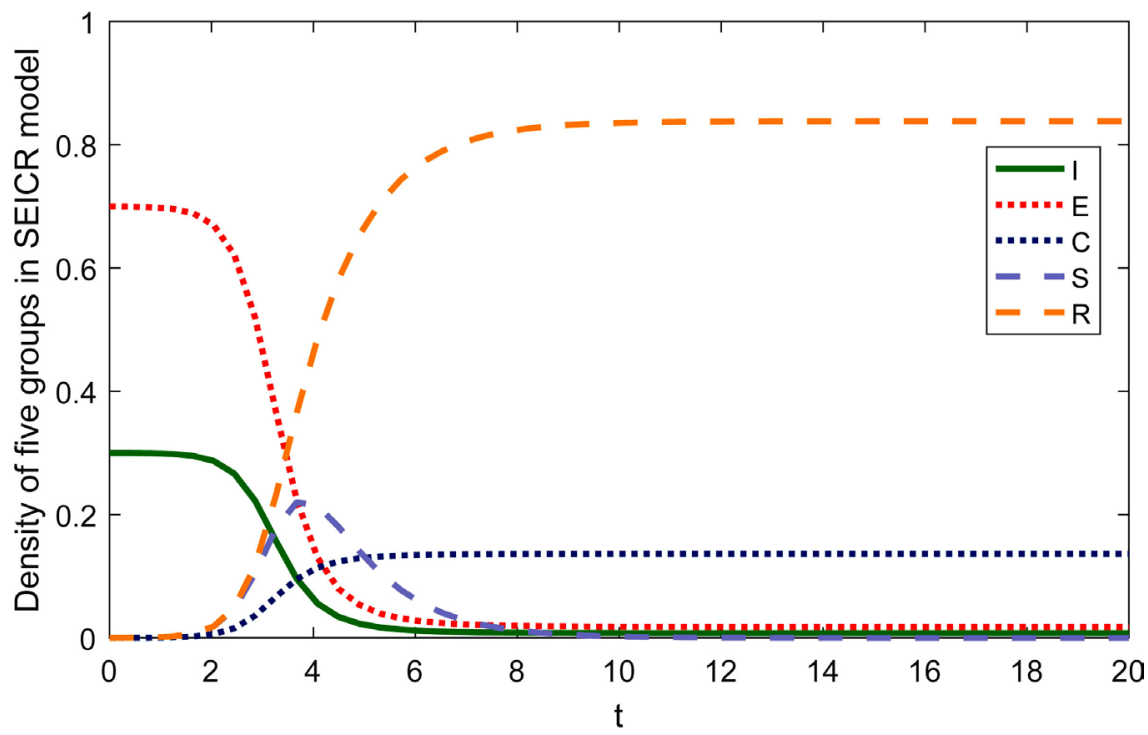

Figure 2. Density of spreaders, educatees, ignorants, counters and recovereds over time, with $\alpha_{1}=\beta_{1}=0.5, \alpha_{2}=\beta_{2}=0.4, g=0.2, \eta=0.1, \epsilon=0.5$. 
always decreases, and eventually evolve to a stable value.

Figures 3-5 show how the densities of spreaders change over time for different system parameters include $\epsilon, \alpha_{1}, \beta_{1}, \alpha_{2}, \beta_{2}, g$ and $\eta$, and the change is consistent with theorem 2. It is interest that the higher parameter $\beta_{1}, \beta_{2}$, the earlier the outbreak, the larger the peak of the outbreak, but the shorter the outbreak period (Figure 4). However, if the parameter $X$ is lowered, the outbreak period will not come earlier, and the outbreak period will be longer (in Figure 5).

\section{Conclusion}

In this paper, considering the social education level and the counterattack mechanism,

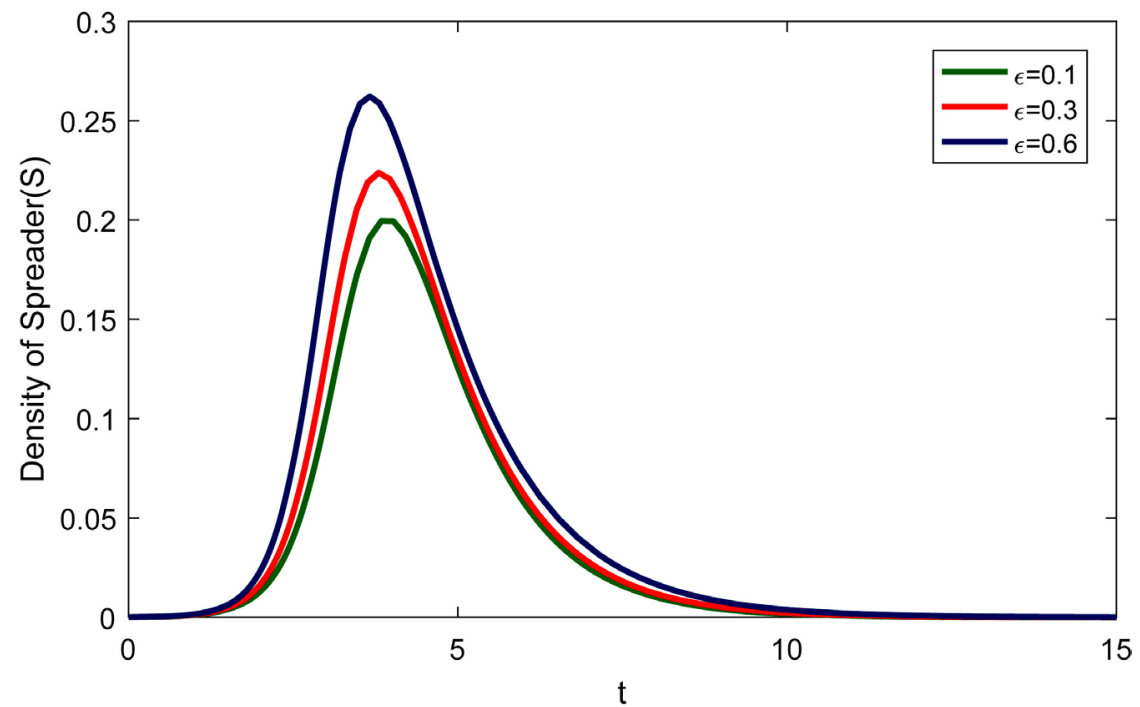

Figure 3. Density of spreaders over time under different population's education rate $\epsilon$, with $\alpha_{1}=\beta_{1}=0.5, \alpha_{2}=\beta_{2}=0.4, g=0.2, \eta=0.1$.

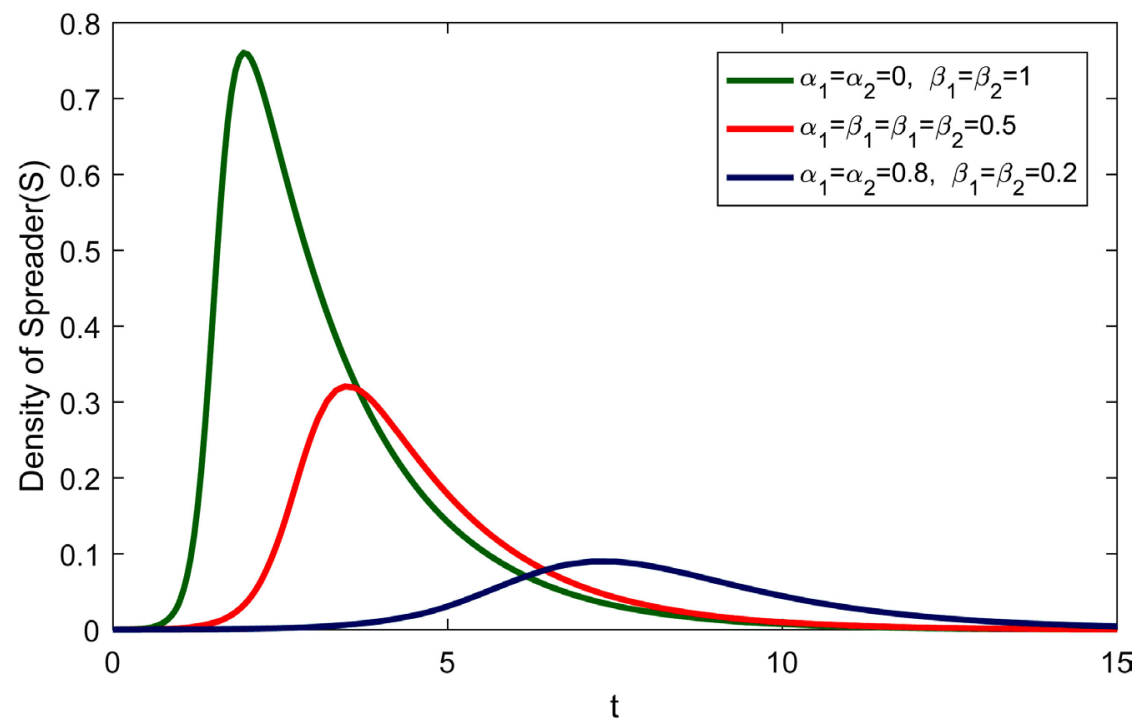

Figure 4. Density of spreaders over time under different $\alpha_{1}, \beta_{1}, \alpha_{2}, \beta_{2}$, with $g=0.2, \eta=0.1, \varepsilon=0.5$. 


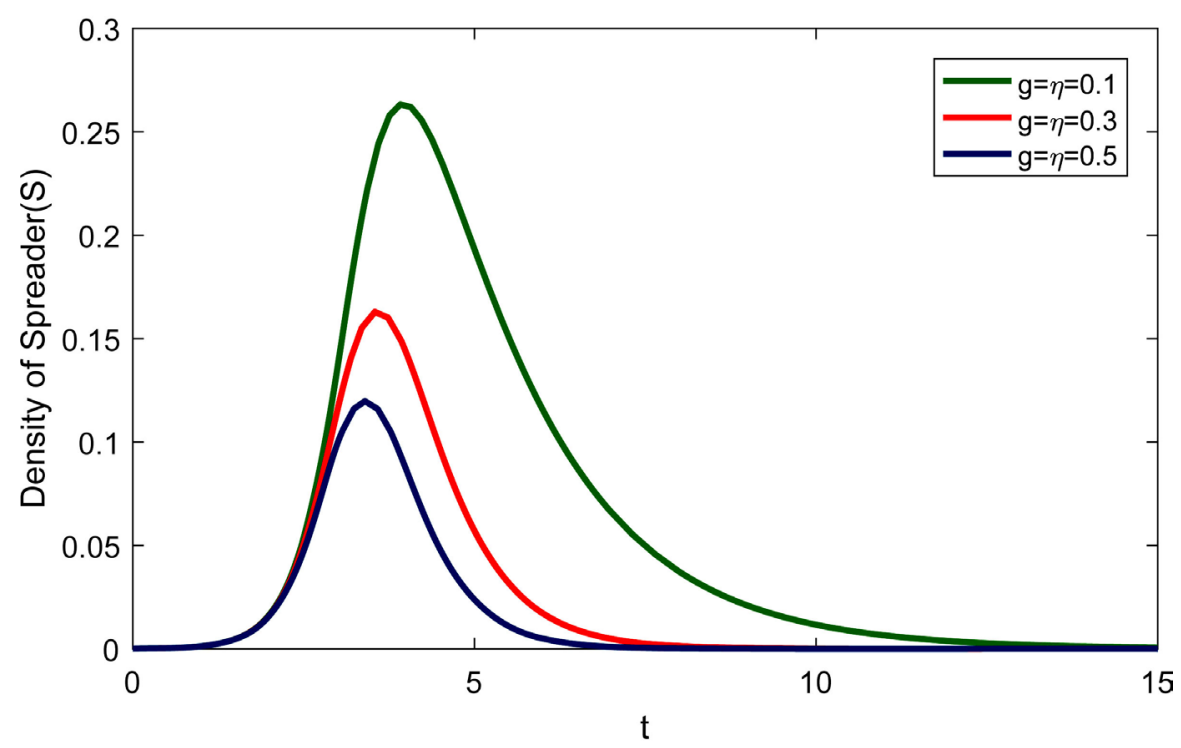

Figure 5. Density of spreaders over time under different $g, \eta$, with $\alpha_{1}=\beta_{1}=0.5, \alpha_{2}=\beta_{2}=0.4, g=0.2, \eta=0.1, \epsilon=0.5$.

we analyze the dynamics of rumor propagation. The results of simulations show that improving education level and increasing the ratio of counter are effective in reducing the risk of rumor propagation and enhancing the resistance to rumor propagation.

\section{Acknowledgements}

The research has been supported by The National Natural Science Foundation of China (11561004) and The 12th Five-year Education Scientific Planning Project of Jiangxi Province (15ZD3LYB031).

\section{Conflicts of Interest}

The authors declare no conflicts of interest regarding the publication of this paper.

\section{References}

[1] Peterson, W.A. and Gist, N.P. (1951) Rumor and Public Opinion. American Journal of Sociology, 57, 159-167. https://doi.org/10.1086/220916

[2] Kesten, H. and Sidoravicius, V. (2005) The Spread of a Rumor or Infection in a Moving Population. Annals of Probability, 33, 2402-2462.

https://doi.org/10.1214/009117905000000413

[3] Kostka, J., Oswald, Y.A. and Wattenhofer, R. (2008) Word of Mouth: Rumor Dissemination in Social Networks. International Colloquium on Structural Information \& Communication Complexity, Villars-sur-Ollon, 17-20 June 2008, 185-196. https://doi.org/10.1007/978-3-540-69355-0_16

[4] Ganesh, A.J., Kermarrec, A.M. and Massoulie, L. (2002) Hiscamp: Self-Organizing Hierarchical Membership Protocol. Workshop on ACM Sigops European Workshop. https://doi.org/10.1145/1133373.1133398 
[5] Thomas, S.A. (2007) Lies, Damn Lies, and Rumors: An Analysis of Collective Efficacy, Rumors, and Fear in the Wake of Katrina. Sociological Spectrum, 27, 679-703. https://doi.org/10.1080/02732170701534200

[6] Bhavnani, R. and Kuklinski, J.H. (2009) Rumor Dynamics in Ethnic Violence. Journal of Politics, 71, 876. https://doi.org/10.1017/S002238160909077X

[7] Galam, S. (2002) Modelling Rumors: The No Plane Pentagon French Hoax Case. Physica A: Statistical Mechanics \& Its Applications, 320, 571-580.

[8] Kimmel, A.J. (2004) Rumors and Rumor Control: A Manager's Guide to Understanding and Combatting Rumors. Lawrence Erlbaum Associates.

[9] Daley, D.J. and Kendall, D.G. (1965) Stochastic Rumours. IMA Journal of Applied Mathematics, 1, 42-55. https://doi.org/10.1093/imamat/1.1.42

[10] Daley, D.J. and Gani, J. (1999) Epidemic Modelling: An Introduction. Cambridge University Press, Cambridge. https://doi.org/10.1017/CBO9780511608834

[11] Maki, D.P. and Thompson, M. (1973) Mathematical Models and Applications: With Emphasis on the Social, Life, and Management Sciences. Prentice Hall, Upper Saddle River.

[12] Pittel, B. (1990) On a Daley-Kendall Model of Random Rumours. Journal of Applied Probability, 27, 14-27. https://doi.org/10.2307/3214592

[13] Newman, M.E., Forrest, S. and Balthrop, J. (2002) Email Networks and the Spread of Computer Viruses. Physical Review E, Statistical, Nonlinear, and Soft Matter Physics, 66, Article ID: 035101. https://doi.org/10.1103/PhysRevE.66.035101

[14] Sudbury, A. (1985) The Proportion of the Population Never Hearing a Rumour. Journal of Applied Probability, 22, 443-446. https://doi.org/10.2307/3213787

[15] Holger, E., Lutz-Ingo, M. and Stefan, B. (2002) Scale-Free Topology of E-Mail Networks. Physical Review E, Statistical, Nonlinear, and Soft Matter Physics, 66, Article ID: 035103.

[16] Smith, R.D. (2002) Instant Messaging as a Scale-Free Network.

[17] Fang, W., Yamir, M. and Yaoru, S. (2006) Structure of Peer-to-Peer Social Networks. Physical Review E Statistical Nonlinear \& Soft Matter Physics, 73, Article ID: 036123.

[18] Zanette, D.H. (2001) Critical Behavior of Propagation on Small-World Networks. Physical Review E Statistical Nonlinear \& Soft Matter Physics, 64, Article ID: 050901. https://doi.org/10.1103/PhysRevE.64.050901

[19] Zanette, D.H. (2002) Dynamics of Rumor Propagation on Small-World Networks. Physical Review E Statistical Nonlinear \& Soft Matter Physics, 65, Article ID: 041908. https://doi.org/10.1103/PhysRevE.65.041908

[20] Yamir, M., Maziar, N. and Alessandro, V. (2004) Efficiency and Reliability of Epidemic Data Dissemination in Complex Networks. Physical Review E Statistical Nonlinear \& Soft Matter Physics, 69, Article ID: 055101.

[21] Moreno, Y., Pastorsatorras, R. and Vespignani, R. (2001) Epidemic Outbreaks in Complex Heterogeneous Networks. The European Physical Journal B Condensed Matter and Complex Systems, 26, 521-529. https://doi.org/10.1140/epjb/e20020122

[22] Nekovee, M., Moreno, Y., Bianconi, G. and Marsili, M. (2008) Theory of Rumour Spreading in Complex Social Networks. Physica A Statistical Mechanics \& Its Applications, 374, 457-470. https://doi.org/10.1016/j.physa.2006.07.017

[23] Zhao, L., Cui, H., Qiu, X. and Wang, X. (2013) Sir Rumor Spreading Model in the New Media Age. Physica A Statistical Mechanics \& Its Applications, 392, 995-1003. 
[24] Zhao, L., Wang, J., Chen, Y., Qin, W., Cheng, J. and Cui, H. (2012) Sihr Rumor Spreading Model in Social Networks. Physica A Statistical Mechanics \& Its Applications, 391, 2444-2453. https://doi.org/10.1016/j.physa.2011.12.008

[25] Zan, Y., Wu, J., Ping, L. and Yu, Q. (2014) Sicr Rumor Spreading Model in Complex Networks: Counterattack and Self-Resistance. Physica A Statistical Mechanics \& Its Applications, 405, 159-170. https://doi.org/10.1016/j.physa.2014.03.021

[26] Afassinou, K. (2014) Analysis of the Impact of Education Rate on the Rumor Spreading Mechanism. Physica A Statistical Mechanics \& Its Applications, 414, 43-52. https://doi.org/10.1016/j.physa.2014.07.041 